\title{
M.E.Sharpe
}

\section{SOCIAL SKILLS TRAINING IN THE TREATMENT OF NEGATIVE SYMPTOMS}

Author(s): JOHN T. WIXTED, RANDALL L. MORRISON and ALAN S. BELLACK

Reviewed work(s):

Source: International Journal of Mental Health, Vol. 17, No. 1, PERVENTING DISABILITY AND RELAPSE IN SCHIZOPHRENIA: II. Psychosocial Techniques and Working With Families (Spring 1988), pp. 3-21

Published by: M.E. Sharpe, Inc.

Stable URL: http://www.jstor.org/stable/41344481

Accessed: 23/08/2012 00:21

Your use of the JSTOR archive indicates your acceptance of the Terms \& Conditions of Use, available at http://www.jstor.org/page/info/about/policies/terms.jsp

JSTOR is a not-for-profit service that helps scholars, researchers, and students discover, use, and build upon a wide range of content in a trusted digital archive. We use information technology and tools to increase productivity and facilitate new forms of scholarship. For more information about JSTOR, please contact support@jstor.org. 


\title{
SOCIAL SKILLS TRAINING IN THE TREATMENT OF NEGATIVE SYMPTOMS
}

\author{
JOHN T. WIXTED, RANDALL L. MORRISON, \\ AND ALAN S. BELLACK
}

Deficits in interpersonal functioning have been associated with schizophrenia since the disorder was first described. These deficits can be pervasive and often persist even when the more disabling psychotic symptoms have been pharmacologically controlled [1]. Although overt psychosis is generally the most salient and disturbing feature of schizophrenia, the importance of social dysfunction cannot be overemphasized. The results of several large-scale investigations have demonstrated that poor premorbid functioning, especially in the area of social relationships, is prognostic of poor long-term outcome [2-4]. In addition, studies have consistently found that schizophrenic patients whose family relationships are characterized by hostile interpersonal interactions are especially likely to require rehospitalization within nine months of discharge [5,6]. In view of these findings, treatments aimed at teaching schizophrenic patients more effective social skills have become a standard component of many psychiatric rehabilitation programs [7]. Indeed, the combined application of neuroleptic therapy to control psychotic symptoms and social skills training to remedy chronic interpersonal dysfunction is now regarded as one of the most promising approaches to the overall treatment and management of schizophrenia [8].

In recent years the distinction between the lingering deficit symptoms of schizophrenia and the more transitory psychotic symptoms of the disorder has been the subject of considerable theoretical speculation and empirical inquiry [1,9-11]. The purpose of this article is to

The authors are associated with the Medical College of Pennsylvania at Eastern Pennsylvania Psychiatric Institute, 3200 Henry Ave., Philadelphia, PA 19129. 
bring into sharper focus the relationship between recent conceptual advances pertaining to the symptomatology of schizophrenia and social skills training. Of particular interest is the role of social skills training in helping to alleviate what have become known as the "negative symptoms" of schizophrenia.

Contemporary interest in the subtyping of schizophrenic symptoms was sparked by Strauss and associates [1]. They proposed a tripartite framework in which they distinguished among positive symptoms, negative symptoms, and "disorders in relating." Positive symptoms consist of the manifest psychotic features of the disorder that clearly exceed the boundaries of normal experience. Hallucinations and delusions are the most commonly cited examples of these symptoms. Negative symptoms, on the other hand, are characterized by the loss or absence of normal functioning, and include such phenomena as apathy, blunting of affect, and thought-blocking. These symptoms, in particular, have commanded a great deal of attention in recent years because they have been hypothesized to be inflexible, indicative of intellectual impairment, prognostic of poor outcome, and resistant to neuroleptic therapy [10-12].

The third symptom category identified by Strauss and associates, disorders in relating, comprises the social and interpersonal deficits of schizophrenia discussed above. A few investigators continue to refer to this as a separate symptom cluster, but most have now adopted a strictly dichotomous view of schizophrenic symptoms (positive vs. negative). Some have incorporated aspects of Strauss and associates' third category into their definition of negative symptoms (e.g., Andreasen [9]), while others have rejected the notion that impaired social competence is a direct reflection of the schizophrenia disease process (e.g., Crow [10]). As a result of these differing views, definitions of the negative symptom construct vary widely among investigators studying schizophrenia.

Despite this disagreement, patients who exhibit negative symptoms (however defined) have been consistently found to be more socially impaired than other schizophrenic patients. For example, Andreasen \& Olsen [13], using a broad-based definition of negative symptoms that included a measure of social withdrawal, found that patients with negative symptoms had poorer premorbid adjustment and greater so- 
cial impairment than those who exhibited predominantly positive, or a mixture of both positive and negative, symptoms. Pogue-Geile \& Harrow [14], whose definition of negative symptoms was narrower and did not contain an index of social functioning, reported virtually the same result. Thus, whether or not deficits in social functioning are considered to be part of the symptom construct, schizophrenic patients with negative symptoms appear to be among the most socially impaired of the mentally ill.

Interest in the development of effective treatment strategies for these schizophrenics has accelerated as the severity of the disorder has become apparent $[11,15]$. Opinions vary widely on the merits of neuroleptic medication for this purpose, perhaps because of the multiple definitions the negative symptom construct has received. Based on his review of the literature, Crow [10] argued that negative symptoms are generally unresponsive to antipsychotic drugs. Goldberg [16] reached the opposite conclusion and further claimed that it was the "organiclike" symptoms (e.g., olfactory hallucinations) that were unresponsive to treatment. On the basis of reviews of more recent literature, Meltzer, Sommers, \& Luchins [17] and Schooler [8] also determined that antipsychotic drugs could be effective in the treatment of negative symptoms, especially if the patient were treated during the early stages of the illness.

Although these interpretations are encouraging, Schooler [8] has observed that neuroleptic medication provides only a partial answer to the treatment of schizophrenics with negative symptoms and that the combined use of pharmacological and psychosocial therapies may be the most efficacious approach. Because of the pronounced social impairment exhibited by these patients, social skills training appears to be a natural choice of treatment.

Research concerning the etiology of social impairment in schizophrenics with negative symptoms is lacking, but one possibility is that the chronic nature of negative symptoms consistently interferes with the experiences required to learn effective social behavior. Positive symptoms, which tend to be more transient and episodic, may interfere with these experiences on a more intermittent basis and therefore be less socially damaging. According to this view, social skills training may be especially appropriate for schizophrenics with negative symp- 
toms, who, perhaps more than other psychiatric patients, require explicit training in behavior normally shaped by one's social environment.

The scenario presented above is speculative, and other explanations for the social impairment of schizophrenics with negative symptoms are equally plausible. For example, negative symptoms have been found to be associated with intellectual deficits $[12,13]$. Therefore, in addition to lacking socially relevant experience, schizophrenics displaying such symptoms may lack the capacity to benefit from such experience. Until these questions have been answered empirically, however, teaching schizophrenics social skills appears to be a logical adjunct to pharmacological therapy. We here describe a procedure for social skills training, with particular emphasis on its application to the interpersonal deficits associated with negative symptoms.

\section{Assessment of social skills}

The philosophy of social skills training is that interpersonal abilities are acquired through learning and that skill deficits can be defined, measured, and corrected by appropriate training procedures [18]. A person who lacks interpersonal skills may never have learned them or, in the case of prolonged hospitalization, may have lost them because of disuse [7]. One implication of this perspective is that skill deficits are often situation-specific-that is, a person may have learned how to interact skillfully in some social situations, but not in others. These two principles-that interpersonal skills are learned abilities and that they exhibit situational specificity - distinguish the behavioral model of social skills training from models based on personality traits and the psychodynamics of interpersonal functioning.

The clinical efficacy of social skills training has been empirically demonstrated in numerous single-case [19-22] and group-design studies $[23,24]$. Because some of these studies have dealt with vaguely defined "psychiatric" patients and none with selectively targeted schizophrenics with negative symptoms, additional research will be needed to determine whether the lack of social competence associated with such symptoms can be corrected through skills training. Fortunately, a variety of assessment methods, both formal and informal, have been developed to help identify learning-based skill deficits. These methods 
may be used clinically to select schizophrenics who stand the best chance of benefiting from social skills training.

\section{Selection of patients}

A patient who exhibits deficits in interpersonal functioning is by definition a potential candidate for social skills training. Such patients can be identified through personal interview, interviews with significant others, direct observation of social interaction, or even through chart review.

An important question to be answered before the patient is exposed to skills training is whether the observed difficulties represent a skills deficit or are instead a manifestation of factors unrelated to the patient's learning history. To the extent that negative symptoms interfere with experience required for normal social development [14], social skills training would be appropriate. However, as noted by Carpenter, Heinrichs, \& Alphs [25], negative symptoms can affect social performance for a variety of other reasons, not all of which would warrant the use of skills training. These authors suggest, for example, that certain "primary" negative symptoms may be a reflection of structural brain impairment [10], in which case social skills training may prove to be relatively ineffective.

Although no straightforward assessment procedure can answer the question of whether the social dysfunction associated with negative symptoms reflects a skills deficit, Carpenter and associates [25] have developed a useful decision-tree approach to the problem. Because negative symptoms may coincide with the exacerbation of positive symptoms, the first question to consider is whether the patient is actively psychotic. If so, then all of the patient's symptoms may respond satisfactorily to neuroleptic medication or may remit spontaneously with the passage of time.

If the patient is not actively psychotic, the possibility that negative symptoms are drug-induced should be considered. Akinesia and sedation are two common side effects of drugs that can masquerade as negative symptoms and severely impair social performance. If the negative symptoms persist in spite of efforts to correct these problems, the possibility that the patient's affective state is contributing to the 
symptom picture should be evaluated. Both severe social anxiety and depression can produce behavioral effects that are difficult to distinguish from negative symptoms. Therefore, if an affective or anxiety disorder is suspected, treatments aimed at these problems should probably be administered before skills training is undertaken.

According to the behavioral model of social skills described earlier, skill deficits will tend to be situation-specific. The possible situational specificity of social impairment in schizophrenics with negative symptoms has been a largely unexplored area [26]. Nevertheless, for a skills training program, the identification of situational fluctuations in social performance is essential in order to establish the existence of a skills deficit and to tailor training to the needs of individual patients. Some patients, for example, may have difficulty conversing with peers, but not with family members. Others may adopt a blank facial expression when interacting with members of the opposite sex, but do so to a lesser extent when interacting with persons of the same sex. If a patient exhibits social dysfunction uniformly across all situations, an alternative explanation for those symptoms (e.g., depression) should be reconsidered.

In summary, if a patient displays some situational variability in social performance and is not actively psychotic, hampered by side effects of drugs, or suffering from severe anxiety or depression, social skills training would appear to be an appropriate intervention.

\section{Areas of skill deficits}

The deficits that a patient exhibits in different social situations must be defined in detail if training is to be successful. Vague descriptions of interpersonal difficulties suggest few if any behaviors to target for change. Table 1 lists the range of behavior that is usually evaluated in this regard. At the most elementary level, the components of social skill can be divided into various verbal, nonverbal, and social perceptual abilities.

Expressive elements consist of the verbal and nonverbal response parameters involved in communicating a message to another person. The most important of these is speech content, a skill that can be 
Table 1

\section{Components of Social Skill}

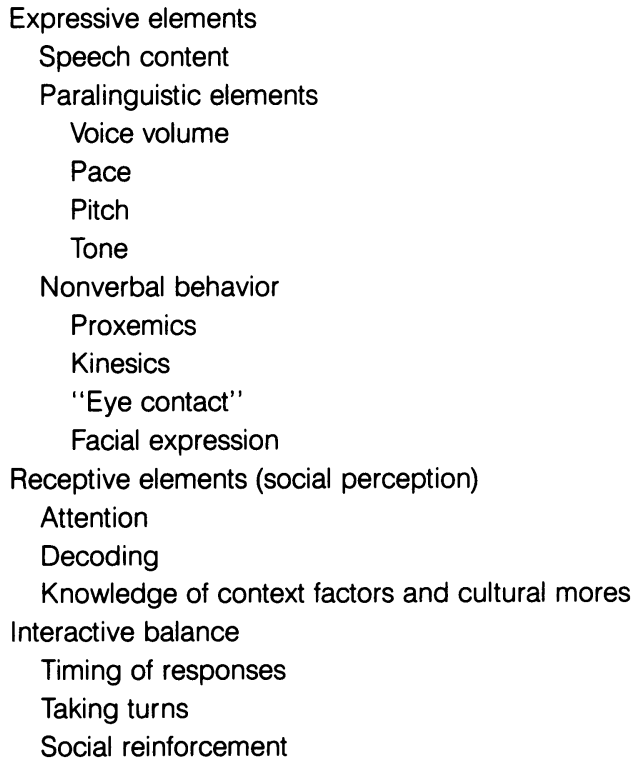

severely compromised in patients with negative symptoms. The words one chooses to use are of obvious importance in determining the meaning of a spoken message. Thus, poverty of speech and/or poverty of content of speech can severely impair social performance. These symptoms, which constitute part of the negative symptom construct by most definitions, are common targets of skills training programs.

Paralinguistic elements refer to the voice parameters that serve to qualify a verbal message. The same verbal statement can assume different shades of meaning depending on whether it is spoken in a flat or an animated tone, loud or soft voice, or slow or rapid manner. Psychiatric patients often exhibit deficits in this area, especially with respect to voice tonality. Indeed, the most universally accepted negative symptom 
of schizophrenia, blunted affect, consists in part of monotone speech. Because deficits such as these can severely impair social performance, social skills training commonly includes direct training in appropriate use of voice parameters.

Nonverbal behavior refers to a person's bodily positions and movements during social interaction. As with paralinguistic elements, these responses play an important role in that they can either strengthen or detract from a verbal message. Thus, for example, an assertive refusal of an unreasonable request is more likely to be effective when accompanied by direct eye contact and a serious facial expression than when accompanied by downcast eyes and shuffling feet.

Once again, many of the deficits in this category of social behavior also comprise part of the negative symptom construct. These include diminished facial responsiveness, minimal eye contact, and restricted bodily movements and gestures (kinesics).

Receptive elements refer to a person's social perceptual abilities. Effective social interaction depends on the ability to detect, interpret, and respond appropriately to what are often subtle interpersonal cues. For example, patients may need to be taught that conversational partners will sometimes indirectly signal that they are ready to end a conversation (e.g., by glancing at the door) and that steps taken to end the conversation at this point are appropriate.

An important prerequisite of this ability, and one that can easily be taken for granted, is focused attention. Attentional deficits are common in schizophrenic patients [27-29], and they may be particularly severe in patients who exhibit negative symptoms $[9,30]$. Thus, many patients will require extensive training to attend to relevant interpersonal cues. In addition to simply attending to these cues, most patients will need to learn how to respond appropriately to them. Appropriate responses are determined both by transient contextual factors and by cultural mores. Because of the intellectual impairment associated with negative symptoms [12], patients' knowledge of these factors may be quite limited before training.

A final category of social skill is interactive balance. Included in this category are timing responses, taking turns, and providing social reinforcement. Psychiatric patients may, for example, exhibit inappropri- 
ately long response latencies when they are asked a question, causing others to feel uncomfortable. They may also be ignorant of the giveand-take of conversational interaction and try to inject statements at inopportune moments. Social reinforcement refers to the cues a person provides to another to indicate attention and interest (e.g., head nodding, "uh-huh," occasional smiles). The absence or infrequent use of responses such as these is characteristic of patients who are said to exhibit negative symptoms.

The individual responses described above constitute many of the important components of social skill. They are defined on the most elementary level to permit detailed specification of any deficits that may exist. However, considered on that level alone, behavior does not fully capture the essence of skillful social performance. Competent social performance is characterized not only by the correct execution of individual response elements but also by the integration of those elements into a variety of high-level or complex behavioral repertoires. Conversation skills, assertive skills, heterosocial skills (e.g., dating behavior), and vocational skills are all examples of the complex repertoires that constitute skillful social behavior.

Schizophrenic patients may exhibit deficits in all of these areas, and all have been the target of social skills training programs for these patients [31]. A detailed treatment manual for training psychiatric patients in the use of these skills has been developed by Beidel and colleagues [32].

In order to train a patient in the use of complex behavioral skills, those skills must be reduced to less complex component responses. As an example, consider training in basic conversational skills. Table 2 lists some of the behavioral skills required to carry on an ordinary conversation. Separate abilities are required for initiating, maintaining, and ending a conversation. However, even at that level of specificity, the moment-by-moment responses required of the patient are not specified. Table 3 provides an even more detailed description of the behavioral components of one aspect of conversation skills (initiating a conversation). Only when reduced to this level of detail can a patient be trained in the use of the responses listed in Table 1 as they relate to conversational abilities. A similar pro- 
Table 2

\section{Conversational Skills}

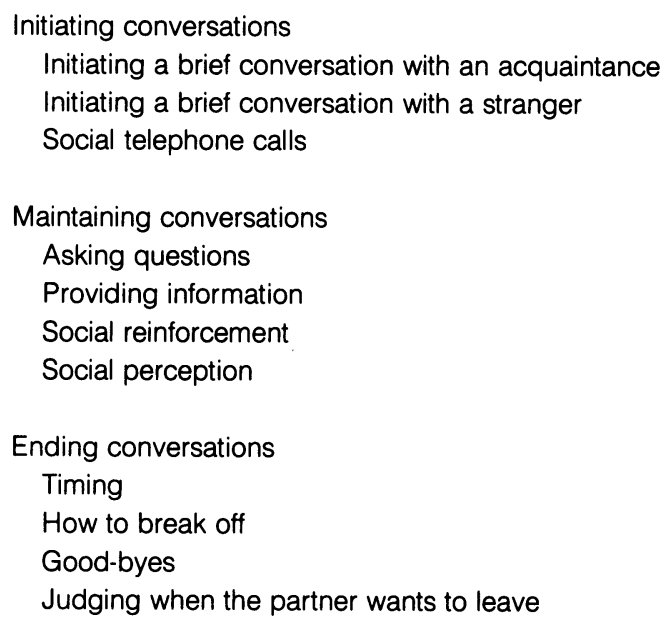

Table 3

\section{Initiating a Conversation}

1. Make contact. e.g., Smile and say, "Hello, (Name)."

2. Ask a general question. e.g., "How have you been?"

3. Ask a specific question or answer a question from the other person.

4. Give a reason for leaving, and say good-bye e.g., "I have an appointment..." 
cedure would be followed for training assertive, heterosocial, and job skills [32].

\section{Assessment procedures}

Behavioral observation. The best way to determine whether a patient exhibits deficits in any of the areas described above is through extended observation of his or her social behavior. To this end, clinical staff can be trained to attend specifically to relevant social responses. A nurse's observations of a patient's conversational abilities, for example, can be invaluable in the development of an individualized skills training program. However, because the hospital environment does not provide a full range of social opportunities, additional assessment methods are usually necessary. Were it not for the time and expense involved, a very complete social assessment might be provided by direct observation of the patient's behavior in his or her natural environment. Fortunately, several more cost-effective methods have been developed in recent years. The most widely employed procedure in this regard is the "role-play" test.

Role-play tests. A role-play test involves the brief enactment of a social interaction as if the scene were really happening. In a typical test, a situation is described to the patient, and the therapist, playing the role of another person in the scene, issues a verbal prompt. The patient is instructed to respond to the prompt as realistically as possible, and the therapist extends the interaction for one or two more interchanges. For example, a scene description read by the therapist might be as follows: "Imagine you are home watching television when your roommate walks in and changes the channel without asking. He says, 'Let's watch this channel for a while.' ' Following the patient's response, the therapist might continue: "You've been watching your shows all day. This one is better anyway." Once again, the patient would be asked to respond to the therapist's prompt.

Several role-play interactions can be videotaped for later scoring in order to assess the patient's behavior in a variety of situations. Alternatively, the clinician can simply evaluate the patient's role-play performance on an ongoing basis, attending in particular to the skills listed in Table 1. 
Bellack [33,34] has analyzed and reviewed the empirical evidence pertaining to the validity and utility of this assessment procedure. Although the data do not provide conclusive support for this method, he nevertheless concludes that role-play tests may be the best option available for direct observational assessment.

Rating scales. Behavioral rating scales are another commonly employed assessment procedure that may be used as an adjunct to roleplay assessment. These instruments offer a convenient method for obtaining a quantitative measure of the behavior of interest.

Three negative symptom rating scales that have been shown to possess adequate psychometric properties are the Rating Scale for Emotional Blunting [35], the Scale for the Assessment of Negative Symptoms [9], and a hybrid scale derived from the Nurses' Observation Scale for Inpatient Evaluation and the Schedule for Affective Disorders and Schizophrenia [36]. Although these measures are employed primarily for research purposes, the information they provide regarding the nature and extent of negative symptoms suggests that they can be useful to the clinician as well.

The assessment procedures described in this section are designed to select appropriate patients for a skills training program, define their skill deficits as accurately as possible, and measure those deficits before and throughout training. Application of these procedures will help prevent the inappropriate use of social skills training and alert the therapist to ongoing progress or lack thereof.

\section{Social skills training procedures}

Social skills training is an educative or reeducative process that is more like motor skills training (e.g., teaching someone to play tennis) than traditional psychotherapy. The therapist assumes the role of a teacher who instructs the patient in the use of social skills and demonstrates how they are applied. In order to learn these skills, patients are required to practice newly acquired responses until they are able to perform them adequately. In general, an adequate level of performance is simply the minimal acceptable response, not the epitome of social skill.

Social skills training for psychiatric patients is generally conducted 
in a group, and proceeds in a highly structured fashion, as described below. A typical group consists of approximately eight patients, and meets for an hour three times a week. Fewer meetings would be unlikely to foster significant behavioral change, and much more could exceed the learning capacity of many psychiatric patients (although Wallace [36] has used much more intensive training with good results). The duration of training will vary as a function of the syllabus. Usually, a minimum of one month must be devoted to a particular content area (e.g., assertiveness, or job interview skills).

The groups are best conducted by two therapists, as it is difficult for one person to teach social skills and maintain constant control of the group. Moreover, the use of two therapists can greatly facilitate the demonstration, or modeling, of new social skills.

In order to organize and structure group activities, the therapist must identify a set of social situations that the patients typically encounter and that are relevant to their individual skill deficits. For example, initiating and maintaining conversations with other patients and requesting help from family members might be two situations around which to work on individual skills. Although the therapist will probably have to create most of these scenes, the patients in the group may be invited to describe situations in which they experience difficulty.

The course of training generally proceeds from teaching the requisite skills for the least challenging social situation to the most challenging, using as many sessions as necessary at each level. The rationale for proceeding in this manner is that skills are more easily acquired under less threatening conditions, and the skills mastered at one level can serve as the basis for more complex skills to be learned later. Five techniques are generally employed to teach the patient a specific skill: instructions, modeling, rehearsal (role-playing), feedback and positive reinforcement, and homework.

\section{Instructions}

When presenting a new skill to be learned, the first step is to instruct the patient in its use and to provide a rationale for learning to use it. For example, when addressing the common negative symptom of blank facial expression, the therapist might say, "When listening to another 
person talk to you, an occasional nod and smile will let that person know that you are listening and are interested in what he has to say." In keeping with the emphasis on training to achieve the minimally acceptable response, the instructions should be simple and straightforward and should not attempt to address subtle nuances of every skill.

\section{Modeling}

Although verbal instructions are helpful, one of the best ways to communicate essential information about a skill is simply to demonstrate or model its use. Immediately before demonstrating a particular skill, the therapist should draw attention to the most important response component (e.g., "Notice how I sometimes nod while I am listening"). A brief interaction can then be arranged, calling on the co-therapist (if available) or a patient to assist in the demonstration. The enactment should be brief and to the point. Extended demonstrations are likely to reduce attention or draw the patients' attention to extraneous behavior. The skill may have to be demonstrated repeatedly for some patients.

\section{Role-playing}

After the skill has been demonstrated, the patient is asked to try to mimic the therapist's behavior in a brief role-play interaction. This is a most important component of social skills training, as simply talking about and/or viewing skillful behavior is unlikely to impart the target skills to the patient. As in the scenario used to model the skill, each patient attempts to apply the skill in role-play with the therapist.

Once the patients have mastered the minimal components of the skill, the therapist can supervise further role-play and practice between the patients themselves. Although the learning abilities of individual patients vary, in most cases extended practice and repetition will be necessary.

\section{Feedback and social reinforcement}

In an effort to shape appropriate social skills, the therapist should provide feedback and positive reinforcement following every role-play. The feedback should be specific and focus initially on the positive 
aspects of the response under training. This principle is adhered to even when the patient's performance is grossly deficient. Only after appropriately praising the patient's performance does the therapist suggest changes. For example, the therapist may say, "You did a very good job of looking at my eyes when you first started to speak. This time, try to do even more of that."

A patient who experiences frequent feelings of success and who receives prodigious praise and encouragement from the therapist is far more likely to retain the motivation required to practice social skills to proficiency than one who receives only suggestions for improvement.

\section{Homework}

Role-play interaction provides an opportunity for the patient to learn and practice new skills, but additional practice between sessions is required in order for those skills to generalize to other settings. Therefore, patients are routinely given homework assignments to use the skills acquired in a particular session with other people before the next session. Specific assignments such as "Ask your roommate to help you play cards tonight" are more likely to meet with compliance than vague assignments such as "Try requesting things from people."

The assignment should be one that is likely to meet with success: the therapist should be reasonably certain that the patient is capable of carrying out the assignment and that it stands a good chance of receiving a favorable response. At the beginning of each session, the homework assignments from the preceding session are reviewed, and any problems that have arisen are resolved before proceeding to a new skill.

\section{Social perception training}

Mastery of individual and even complex response skills does not guarantee their effective use in social situations. In addition to training in overt response skills, patients usually must be taught when and where to use them. These abilities require that the patient attend to and correctly interpret both interpersonal and contextual cues. For example, a patient who has just mastered the ability to initiate a conversation 
may also need to learn that it can be unwise to exercise that skill with someone displaying an extremely unreceptive facial expression. Similarly, a patient who has learned to effectively refuse a request may need to learn that it is appropriate to do so when dealing with a salesperson, but not when dealing with a police officer.

Training in social perceptual abilities does not follow a separate structured sequence of activities, but is instead integrated into the response training procedures described above. The objective is to train the patient to attend to and interpret interpersonal cues that signify the feelings and motives of other people and to be aware of contextual variables that determine the appropriateness of various responses. This can be accomplished during role-play interaction by introducing subtle variations in the therapist's behavior and inquiring about the meanings of those variations. For example, during role-plays involving casual conversation, the therapist can increasingly exhibit nonverbal cues indicating a lack of interest and a desire to leave (e.g., fidget, glance at watch, look at door). After each role-play, patients can be questioned about possible interpretations of and acceptable responses to such behavior. Training with respect to contextual cues is achieved mainly through didactic means. For instance, a portion of each session can be devoted to discussing the social rules that govern acceptable use of the skills under consideration.

The social perceptual abilities of schizophrenics have, unfortunately, been given little empirical attention in the social skills literature $[38,39]$. Although the findings have been inconsistent, some evidence suggests that schizophrenics have marked difficulty identifying emotions displayed nonverbally $[40,41]$. This difficulty may be a consequence of the attentional and cognitive impairment associated with schizophrenia or may reflect an independent deficit. In either case, patients with negative symptoms are likely to exhibit especially marked deficits in these areas, which suggests that social perception training with these patients may progress rather slowly.

Wallace [37] has developed a program specifically intended to improve the information-processing skills of schizophrenic patients. In this program patients are taught to receive and process incoming stimuli accurately and subsequently to send effective verbal and nonverbal responses. The distinctive component of this approach is its emphasis 
on interpersonal stimulus processing, or problem solving, during which patients are taught to generate various response options, weigh the value of those options, and devise an appropriate response strategy. This approach has proven to be effective with many schizophrenic patients, and appears to offer a practical means of addressing the information-processing deficits of schizophrenics with negative symptoms.

\section{Conclusion}

Social skills training has been shown to be an effective means of increasing the social competence of schizophrenic patients, and may also prove to be an effective aid in the treatment of the social deficits associated with the negative symptoms of schizophrenia. Indeed, many of the deficits routinely targeted by skills training programs are now considered to be integral components of the negative symptom construct. Whether a patient who exhibits pronounced negative symptoms will benefit from the addition of social skills training to neuroleptic therapy has not yet been empirically evaluated. It may be that such patients will prove to be resistant to all forms of therapy. Nevertheless, in light of its success in treating such symptoms in the schizophrenic patient population as a whole, social skills training appears to offer one promising approach to alleviating negative symptoms.

\section{Acknowledgment}

Preparation of this manuscript was supported in part by grants $\mathrm{MH}$ 39998 and MH 38636 from the National Institute of Mental Health.

\section{References}

1. Strauss, J. S., Carpenter, W. T., \& Bartko, J. J. (1974) The diagnosis and understanding of schizophrenia. Part III. Speculation on the processes that underlie schizophrenic symptoms and signs. Schizophrenia Bulletin, No. 11, p. 61.

2. Strauss, J. S., Klorman, R., \& Kokes, R. F. (1977) Premorbid adjustment in schizophrenia: Concepts, measures, and implications. Part V: The implications of findings for understanding, research, and application. Schizophrenia Bulletin, 3, 240.

3. Zigler, E., \& Phillips, L. (1961) Social competence and outcome in psychiatric disorders. Journal of Abnormal and Social Psychology, 63, 264.

4. Zigler, E., \& Phillips, L. (1962) Social competence and the process-reactive distinction in psychopathology. Journal of Abnormal and Social Psychology, 65, 215. 
$\rightarrow$ Brown, G. W., Birely, J. L. T., \& Wing, J. K. (1972) Influence of family life on the course of schizophrenic illness. British Journal of Psychiatry, 121, 241.

$\rightarrow$ Vaughn, C. E., \& Leff, J. P. (1976) The influence of family and social factors on the course of psychiatric illness: A comparison of schizophrenic and depressed neurotic patients. British Journal of Psychiatry, 129, 125.

7. Bellack, A. S., \& Hersen, M. (Eds.) (1979) Research and practice in social skills training. New York: Plenum.

8. Schooler, N. R. (1986) The efficacy of antipsychotic drugs and family therapy in the maintenance treatment of schizophrenia. Journal of Clinical Psychopharmacology, 6, 11s.

9. Andreasen, N. C. (1982) Negative symptoms in schizophrenia: Definition and reliability. Archives of General Psychiatry, 39, 784.

$1 \rightarrow$ Crow, T. J. (1980) Molecular pathology of schizophrenia: More than one dimension of pathology? British Medical Journal, 280, 66.

11. Crow, T. J. (1985) The two-syndrome concept: Origins and current status. Schizophrenia Bulletin, 11, 471.

12. Owens, D. G. C., \& Johnstone, E. C. (1980) The disabilities of chronic schizophrenia: Their nature and the factors contributing to their development. British Journal of Psychiatry, 136, 384.

13. Andreasen, N. C., \& Olsen, S. (1982) Negative vs. positive schizophrenia: Definition and validation. Archives of General Psychiatry, 39, 789.

14. Pogue-Geile, M. F., \& Harrow, M. (1984) Negative and positive symptoms in schizophrenia and depression: A follow-up. Schizophrenia Bulletin, 10, 371.

15. Pogue-Geile, M. F., \& Harrow, M. (1985) Negative symptoms in schizophrenia: Their longitudinal course and prognostic importance. Schizophrenia Bulletin, $11,427$.

16. Goldberg, S. C. (1985) Negative and deficit symptoms in schizophrenia do respond to neuroleptics. Schizophrenia Bulletin, 11, 453.

17. Meltzer, H. Y., Sommers, A. A., \& Luchins, D. J. (1986) The effect of neuroleptics and other psychotropic drugs on the negative symptoms of schizophrenia. Journal of Clinical Psychopharmacology, 6, 329.

18. Morrison, R. L., \& Bellack, A. S. (1985) Social skills training. In A. S. Bellack (Ed.), Schizophrenia: Treatment, management, and rehabilitiation. Orlando, FL: Grune \& Stratton. Pp. 247-79.

19. Bellack, A. S., Hersen, M., \& Turner, S. M. (1976) Generalization effects of social skills training in chronic schizophrenics: An experimental analysis. Behaviour Research and Therapy, 14, 391.

20. Edelstein, B. A., \& Eisler, R. M. (1976) Effects of modeling and modeling with instructions and feedback on the behavioral components of social skills. Behavior Therapy, 7, 382.

21. Hersen, M., \& Bellack, A. S. (1976) A multiple-baseline analysis of social skills training in chronic schizophrenics. Journal of Applied Behavior Analysis, 9, 239.

22. Hersen, M., Turner, S. M., Edelstein, B. A., \& Pinkston, S. G. (1975) Effects of phenothiazines and social skills training in a withdrawn schizophrenic. Journal of Clinical Psychology, 34, 588.

23. Bellack, A. S., Turner, S. M., Hersen, M., \& Luber, R. F. (1984) An examination of the efficacy of social skills training for chronic schizophrenic patients. 
Hospital \& Community Psychiatry, 35, 1023.

24. Wallace, C. J., \& Liberman, R. P. (1985) Social skills training for patients with schizophrenia: A controlled clinical trial. Psychiatry Research, 15, 239.

25. Carpenter, W. T., Heinrichs, D. W., \& Alphs, L. D. (1985) Treatment of negative symptoms. Schizophrenia Bulletin, $11,440$.

26. Lewine, R. J., \& Sommers, A. A. (1985) Clinical definition of negative symptoms as a reflection of theory and methodology. In M. Alpert (Ed.), Controversies in schizophrenia: Changes and constancies. New York: Guilford Press. Pp. 267-79.

27. Cromwell, R. L. (1978) Attention and information processing: A foundation for understanding schizophrenia? In L. C. Wynne, R. L. Cromwell, \& S. Matthysse (Eds.), The nature of schizophrenia: New approaches to research and treatment. New York: Wiley. Pp. 219-24.

28. Kornetsky, C., \& Orzack, M. H. (1978) Physiologic and behavioral correlates of attention dysfunction in schizophrenic patients. In L. C. Wynne, R. L. Cromwell, \& S. Matthysse (Eds.), The nature of schizophrenia: New approaches to research and treatment. New York: Wiley. Pp. 196-204.

29. Wohlberg, G., \& Kornetsky, C. (1973) Sustained attention in remitted schizophrenics. Archives of General Psychiatry, 28, 533.

30. Green, M., \& Walker, E. (1984) Susceptibility to backward masking in positive versus negative symptom schizophrenia. American Journal of Psychiatry, $141,1273$.

31. Liberman, R. P., Mueser, K. T., Wallace, C. J., Jacobs, H. E., Eckman, T., \& Massel, H. K. (1986) Training skills in the psychiatrically disabled: Learning coping and competence. Schizophrenia Bulletin, 12, 631.

32. Beidel, D. C., Bellack, A. S., Turner, S. M., Hersen, M., \& Luber, R. F. (1981) Social skills training for chronic psychiatric patients: A treatment manual. JSAS Catalog of Selected Documents in Psychology, 11, 36 (MS. 2257).

33. Bellack, A. S. (1979) A critical appraisal of strategies for assessing social skill. Behavioral Assessment, 1, 157.

34. Bellack, A. S. (1983) Recurrent problems in the behavioral assessment of social skill. Behaviour Research and Therapy, 21, 29.

35. Abrams, R., \& Taylor, M. A. (1978) A rating scale for emotional blunting. American Journal of Psychiatry, 135, 226.

36. Lewine, R. J., Fogg, L., \& Meltzer, H. Y. (1983) Assessment of negative and positive symptoms in schizophrenia. Schizophrenia Bulletin, 9, 368.

37. Wallace, C. J. (1982) The social skills training project of the Mental Health Clinical Research Center for the study of schizophrenia. In J. P. Curran \& P. M. Monti (Eds.), Social skills training: A practical handbook for assessment and treatment. New York: Guilford Press. Pp. 57-89.

38. Morrison, R. L., \& Bellack, A. S. (1981) The role of social perception in social skill. Behavior Therapy, 12, 69.

39. Morrison, R. L., \& Bellack, A. S. The social functioning of schizophrenic patients: Clinical and research issues. Schizophrenia Bulletin. In press.

40. Cutting, J. (1981) Judgement of emotional expression in schizophrenics. British Journal of Psychiatry, 139, 1.

41. Livesay, J. R. (1981) Inconsistent interpersonal judgement in thought-disordered schizophrenia. Psychological Reports, 49, 179. 